\title{
Research on Exhibition Entity Evaluation Model Based on Green Design
}

\author{
Jingjing Chen ${ }^{1}$ Jinchang Chen ${ }^{2}$ \\ ${ }^{1}$ Guangzhou College of South China University of Technology, Guangzhou \\ 510800,China \\ doublejing_nov@126.com \\ ${ }^{2}$ School of Design ,South China University of Technology, Guangzhou 510006,China \\ jcchen@scut.edu.cn
}

\begin{abstract}
The concept of green exhibition design points out that the main forms of exhibition design are entity design and virtual design. The key variable of green exhibition design is entity based on the "4R" principle. A new exhibition entity evaluation model has been proposed based on the energy recovery performance calculation model. It will be more perfect with the functional recovery and recycling energy consumption taken into account. Finally, the paper indicates the exhibition entity recovery situation would be considered by designer in the beginning and the recovery target is the best with the exhibition function being kept.
\end{abstract}

Keywords: Industrial design, Exhibition design, Green design, Entity

\section{Introduction}

Green design is a rather hot topic in recent years. It is defined as "3R", which means reduce, reuse and recycle. The significance of that is to reduce the use of resources, improve the reuse of resources and strengthen the recycling of resources ${ }^{[1]}$. The late 1990s, the "3R" principle develops to the "4R" principle with recover (energy recovery). EU defined the policy for rubbish management according to " $4 \mathrm{R}$ " as reduce $\rightarrow$ reuse $\rightarrow$ recycle $\rightarrow$ re- cover $\rightarrow$ landfill. The policy has positive significance for reducing the energy and energy processing cost ${ }^{[2]}$. It is necessary to integrate with green design and exhibition design to form green exhibition design, which will improve both of them.

The green exhibition design is presented to the audience with the integration of green design and exhibition design. The virtual body could be for multiple used so that there is no resource consumption. Finally, consumption of green exhibition design is the use of entities. This paper proposes a new exhibition entities evaluation model based on the principle of green design according to product assembly time and energy recovery performance calculation model. It achieves the green exhibition of the qualitative and quantitative analysis and optimal design.

\section{The calculation model analysis of product assembly time and energy recovery performance}

There are some research on the use of removable products and reuse of products for energy recovery ${ }^{[3]}$.

- The mode of assembly is i, the mode of disassembly is a and the total number of assembly mode is $\mathrm{n}$;

- The time consuming of assembly mode $\mathrm{i}$ is $\mathrm{T}_{\mathrm{a}}^{\mathrm{i}}$, the average time is $T_{i}$ and the total time is $T$. The 
number of removable products is $\mathrm{N}_{\mathrm{i}}$ and the type number of assembly is L.

- The average time and total time calculation model for product assembly are shown as follows:

$$
\begin{aligned}
T_{i} & =\frac{1}{n} \sum_{a=1}^{n} T_{a}^{i} \\
T & =\sum_{i=1}^{L} T_{i} \times N_{i}
\end{aligned}
$$

- The recyclable performance model is defined according to Eq. (1), Eq. (2) and energy recovery. Set the energy recovery performance as RI, energy cyclic coefficient is $A_{i}$, the weight of the component $\mathrm{i}$ is $\mathrm{W}_{\mathrm{i}}$ and the weight of the entire product is $\mathrm{W}_{\mathrm{p}}$. Then,

$$
R I=A_{i} \times \frac{W_{i}}{W_{p}}
$$

Where, if $\mathrm{RI}=1$, the entire product can be directly recycled without further process. The energy cycle coefficient $\mathrm{Ai}$ is decided according to the definition of the product material properties. It is easy to obtain from a single material, whose values are higher, while it is difficult to obtain from the composite.

The product assembly efficiency and recycling performance can be effectively obtained from the calculation model, but there are three problems:

- Recyclable products need to be demolished in order to reassembly and the disassembly time will be included in the total assembly time;

- Not all components of products can be recycled. Some parts may be recycled totally while others need secondary demolition or more to be recycled. With the increasing in the number of the demolition will reduce the total assembly time and efficiency;
- Not all materials can be recycled. Taking the non-recyclable energy performance into account, it is not worth to recycle some materials needed be processed by chemical reagents, which might cause energy consumption and environment pollution.

It will have great effect on the efficiency of the recovery of recyclable products ignoring such three problems in the evaluation model. It may form a new energy pollution, which adds the pressure of obtaining new resources and go against the original intention of the green exhibition. So, it is necessary to improve the product assembly time and energy recovery performance calculation model.

\section{The improvement of entity exhibi- tion evaluation model}

Based on the "4R" principle of green design, the loss of energy and components during the recycling would be calculated in the model taking the product recovery based on the properties of functions and components modular recycling into account and avoid a forced recovery. In this paper, the quantifiable energy recovery methods and principles are proposed according to the disassembled product recycling:

- The product is modular teardown analysis according to the structure and function of the product,;

- All modules are divided into single parts and component parts. If the component parts can be overall recovery, it would be treated as a single part, or it would be treated as unavailable overall recovery considering double or multiple demolition. It is generally more than the overall recovery, avoiding the repeatedly demolition as far as possible; 
- The energy loss would be taken into account during the double or multiple chemical process of recyclable materials in the recycling;

- Take priority to recover the easy recovery single material components or functional integrity of the overall parts according to the type of material recyclable or non-recyclable. Avoid forced recovery causing the new consumption of resources and environmental pollution.

Then the model to calculate the energy recovery time from disassembled products and recovery performance has been rebuilt: assume the totally time of disassembly and assembly is $\mathrm{T}$, the average demolition time is $T_{a}$, the average assembly time is $T_{b}$, the disassembly number is $\mathrm{n}$, the assembly number is $\mathrm{m}$, the total amount of demolition parts is a, the total amount of assembly parts is $b$, the energy recovery performance is $R$, energy coefficient is $A$, the weight of part is $W_{i}$, the total weight of parts is $\mathrm{W}_{\mathrm{t}}$ and the loss index of recyclable material is $\mathrm{L}$. The formula to calculate the total time of disassembly and assembly is shown as Eq. (4). The formula to calculate the energy index is shown as Eq. (5). (the improvement exhibition entity evaluation model)

$$
\begin{aligned}
T & =\sum_{n=1}^{n} T_{a}+\sum_{m=1}^{m} T_{b} \\
R & =A \times \frac{W_{i}}{W_{t}}-L
\end{aligned}
$$

Where, A refers to the ratio of energy recycling. If $\mathrm{A}=1$, all parts will be recyclable. The energy coefficient of single material is easy to obtain such as metal while it is difficult to obtain the energy coefficient of parts consisting of many kinds of materials.

According to Eq. (4) and Eq. (5), it has better reduce the amount of parts and materials in the beginning of product design and product manufacture. It will decrease the loss of non-recyclable materials and the assembly time. At the same time, it will improve the efficiency of dismantling and recycling parts.

In order to use the entity in green exhibition design, some principles for material and structure have been proposed:

- The full realization of its exhibition function;

- The zero pollution;

- Recycling rate approaching to $100 \%$;

- Reduce the use of entity;

- Reduce the types of materials;

- The product structure is simple.

It is the optimal ideal state when meeting the above six kinds of principles. If the situation can't meet all, take the functional and environmental protection materials into account firstly.

The improvement exhibition entity evaluation mathematical model more emphasis on showing the materials and structures of the entities and the loss is taken into account which makes the model more perfect.

\section{Conclusion}

It means the ethics and responsibility to the community of designers have returned with the widely use of green design. With the development of society, all kinds of designs incorporated with green elements will transfer from "3R" to "4R", which is not only the core method of green design in new era but also the important exhibition design principle.

In this paper, the concept of green exhibition design has been proposed, pointing out that the mainly forms of exhibition design include entity and virtual. Based on the principle of the "4R", green exhibition focuses on entity exhibition finally. The improvement exhibition entity evaluation model has been proposed according to the model to calculate the 
assembly time of entities and the energy recovery performance in recently. Taking the modular recycling by function and material properties and recovery of energy consumption into account makes the evaluation model more rational and complete.

With the development of humanities education and technology, more and more people emphasize on green design. The recovery of exhibition entity should be considered in the beginning of design. At the same time, increase the recovery of all kinds of exhibition entity, reduce the consumption and convert the information completely from exhibition entity. After the exhibition, it converts to the energy of the other product quickly. It will achieve a harmonious society soon in the future.

\section{References}

[1] http://www.hudong.com/wiki/\%E7\% BB\% BF\%Е8\% 89\% B2\%Е8\%AE\%B $\mathrm{E} \% \mathrm{E} 8 \% \mathrm{AE} \% \mathrm{A1}, 2012.1 .4 /$ 2012.1.18

[2] http://baike.baidu.com/view/408575.h tm\#1, 2011.10.19/2012.1.25

[3] Cheng Kun,Wang Junfeng, Methods of Product Disassembly and Green Design[J].Packaging Engineering,2011,1:29-32 